\title{
Finite vector spaces and certain lattices
}

\author{
Thomas W. Cusick \\ 106 Diefendorf Hall, Department of Mathematics, \\ State University of New York at Buffalo, Buffalo, NY 14214-3093 \\ E-mail: cusick@acsu.buffalo.edu
}

Submitted: January 6, 1998; Accepted: March 18, 1998

\begin{abstract}
The Galois number $G_{n}(q)$ is defined to be the number of subspaces of the $n$-dimensional vector space over the finite field $G F(q)$. When $q$ is prime, we prove that $G_{n}(q)$ is equal to the number $L_{n}(q)$ of $n$-dimensional $\bmod q$ lattices, which are defined to be lattices (that is, discrete additive subgroups of n-space) contained in the integer lattice $\mathbf{Z}^{n}$ and having the property that given any point $P$ in the lattice, all points of $\mathbf{Z}^{n}$ which are congruent to $P \bmod q$ are also in the lattice. For each $n$, we prove that $L_{n}(q)$ is a multiplicative function of $q$.
\end{abstract}

Keywords: Multiplicative function; Lattice; Galois numbers; Vector space; Identities 1991 Mathematical Reviews subject numbers: Primary 05A15 05A19 11A25 11H06 Secondary 05A30 94A60 11T99 
THE ELECTRONiC JOURNAL OF COMBINATORICs 5 (1998), \#R17

\section{Introduction}

The well known Gaussian coefficient (or q-binomial coefficient)

$$
\left(\begin{array}{l}
n \\
r
\end{array}\right)_{q}=\frac{\left(q^{n}-1\right)\left(q^{n-1}-1\right) \cdots\left(q^{n-r+1}-1\right)}{\left(q^{r}-1\right)\left(q^{r-1}-1\right) \cdots(q-1)}
$$

is equal to the number of $r$-dimensional vector subspaces of the $n$-dimensional vector space $V_{n}(q)$ over the finite field $G F(q)$. We let $G_{n}=G_{n}(q)$ denote the total number of vector subspaces of $V_{n}(q)$. The numbers $G_{n}$ were named the Galois numbers by Goldman and Rota [4, p. 77].

Goldman and Rota [4] proved the recursion formula

$$
G_{n+1}=2 G_{n}+\left(q^{n}-1\right) G_{n-1}
$$

for the Galois numbers.

Nijenhuis, Solow and Wilf [4] gave a different proof of (1) by using the observation that the $r$-dimensional vector subspaces of $V_{n}(q)$ are in one-to-one correspondence with the $n$ by $n$ matrices over $G F(q)$ which have rank $r$ and are in reduced row echelon form (rref). Recall that such a matrix is in rref if its last $n-r$ rows are all zeros; in each of the first $r$ rows the first nonzero entry is a 1 ; the index of the $i$-th column (called a pivotal column) in which one of these $r$ 1's occurs strictly increases as $i$ increases; and each of these $r$ pivotal columns has only a single nonzero entry. We let $E(r, n, q)$ denote the number of $n$ by $n$ matrices with rank $r$ over the field $G F(q)$ which are in rref. Then it was proved in [4] that

$$
G_{n}(q)=\sum_{r=0}^{n} E(r, n, q)
$$

The correspondence mentioned above gives

$$
E(r, n, q)=\left(\begin{array}{l}
n \\
r
\end{array}\right)_{q}
$$

For example, $E(r, 4,2)$ for $r=0,1,2,3,4$ is $1,15,35,15$ and 1 , respectively.

We shall need the concept of an $n$-dimensional $\bmod q$ lattice, which is defined to be an n-dimensional lattice contained in the integer lattice $\mathbf{Z}^{n}$ and having the special property that given any point $P$ in the lattice, all points of $\mathbf{Z}^{n}$ which are congruent to $P \bmod q$ are also in the lattice. Later in this paper we shall show how the $\bmod q$ lattices are connected to the Galois numbers $G_{n}(q)$. It also turns out that the mod $q$ lattices have an important application in cryptography, which we discuss elsewhere [2]. The set of $\bmod q$ lattices contains various special subsets which can be used in the design of a novel kind of public-key cryptosystem. This idea originated with Ajtai [1]. 
THE ELECTRONiC JOURNAL OF COMBINATORICs 5 (1998), \#R17

\section{The multiplicative property}

We let $L_{m}(q)$ denote the number of $m$-dimensional $\bmod q$ lattices. Our first goal is to prove that $L_{m}(q)$ is a multiplicative function, that is, for any positive integers $r$ and $s$ with $\operatorname{gcd}(r, s)=1$ we have $L_{m}(r s)=L_{m}(r) L_{m}(s)$.

Theorem 1. The function $L_{m}(q)$ is multiplicative for each $m=2,3, \ldots$.

Proof. Clearly, every $m$-dimensional $\bmod q$ lattice is the solution space of some system

$$
A \mathbf{x} \equiv 0 \bmod q
$$

where $A$ is an $m$ by $m$ matrix over the integers mod $q$. Conversely, the solution space of any system (4) is a mod $q$ lattice. (Note that if $\mathbf{e}_{1}, \mathbf{e}_{2}, \ldots, \mathbf{e}_{m}$ is the standard basis for $\mathbf{R}^{m}$, then the $m$ linearly independent vectors $q \mathbf{e}_{i}(1 \leq i \leq m)$ are always solutions of (4), so the solution space is always a lattice of dimension $m$.)

If $\operatorname{gcd}(r, s)=1$, there is a bijection between the set of $m$-dimensional $\bmod r s$ lattices and the set of pairs of $m$-dimensional lattices made up of one mod $r$ lattice and one mod $s$ lattice. The bijection is defined as follows: Given a mod $r s$ lattice which is the solution space of $A \mathbf{x} \equiv 0 \bmod r s$, we associate with it the pair of lattices which are solution spaces of

$$
B \mathbf{x} \equiv 0 \bmod r \text { and } C \mathbf{x} \equiv 0 \bmod s
$$

where the matrices $B$ and $C$ are defined by

$$
A \equiv B \bmod r \text { and } A \equiv C \bmod s
$$

and conversely, given (5) we define a matrix $A$ by (6).

To prove that this is a bijection, we must first show that different lattice pairs give different mod $r s$ lattices. Given relatively prime integers $r$ and $s$, by the definition of $L_{m}(q)$ we can choose two sets of matrices $\left\{B_{i}: 1 \leq i \leq L_{m}(r)\right\}$, where $B_{i}$ is defined over the integers $\bmod r$, and $\left\{C_{i}: 1 \leq i \leq L_{m}(s)\right\}$, where $C_{i}$ is defined over the integers mod $s$, such that every $m$-dimensional mod $r$ lattice is the solution space of exactly one of the systems $B_{i} \mathbf{x} \equiv 0 \bmod r, 1 \leq i \leq L_{m}(r)$, and every $m$-dimensional mod $s$ lattice is the solution space of exactly one of the systems $C_{j} \mathbf{x} \equiv 0 \bmod s, 1 \leq j \leq L_{m}(s)$. Since $\operatorname{gcd}(r, s)=1$, the theory of linear congruences in one variable shows that each pair of simultaneous congruences

$$
A \equiv B_{i} \bmod r, A \equiv C_{j} \bmod s, 1 \leq i \leq L_{m}(r), 1 \leq j \leq L_{m}(s)
$$

defines a unique $m$ by $m$ matrix $A=A_{i j}$, say, over the integers mod $r s$, and these matrices are all different since the pairs $B_{i}, C_{j}$ are. We shall show that the solution spaces (which are the mod $r s$ lattices) of the systems

$$
A_{i j} \mathbf{x} \equiv 0 \bmod r s, 1 \leq i \leq L_{m}(r), 1 \leq j \leq L_{m}(s)
$$

are all distinct. 
THE ELECTRONIC JOURNAL OF COMBINATORICs 5 (1998), \#R17

Let $A_{I J}$ and $A_{K L}$ be any two different matrices chosen from the $A_{i j}$ 's. Then by $(7)$

$$
\left\{\mathbf{x} \bmod r: A_{I J} \mathbf{x} \equiv 0 \bmod r s\right\}=\left\{\mathbf{x}: B_{I} \mathbf{x} \equiv 0 \bmod r\right\}
$$

and

$$
\left\{\mathbf{x} \bmod s: A_{I J} \mathbf{x} \equiv 0 \bmod r s\right\}=\left\{\mathbf{x}: C_{J} \mathbf{x} \equiv 0 \bmod s\right\}
$$

similar equations hold for $A_{K L}$. Since the pairs $B_{I}, C_{J}$ and $B_{K}, C_{L}$ are different, we have either

$$
\left\{\mathbf{x}: B_{I} \mathbf{x} \equiv 0 \bmod r\right\} \neq\left\{\mathbf{x}: B_{K} \mathbf{x} \equiv 0 \bmod r\right\}
$$

or

$$
\left\{\mathbf{x}: C_{J} \mathbf{x} \equiv 0 \bmod s\right\} \neq\left\{\mathbf{x}: C_{L} \mathbf{x} \equiv 0 \bmod s\right\}
$$

so the solution spaces for $A_{I J}$ and $A_{K L}$ are different.

Finally we must show that different mod $r s$ lattices give different lattice pairs. This is clear since each congruence $A \mathbf{x} \equiv 0 \bmod r s$ gives a unique pair of congruences (5), where the matrices $B$ and $C$ are defined by (6).

\section{Counting $\bmod q$ lattices}

Our first goal is to prove explicit formulas for the number of $m$-dimensional mod $q$ lattices, which we denote by $L_{m}(q)$, when $m$ is small.

Theorem 2. The numbers $L_{2}(q)$ and $L_{3}(q)$ are given by

$$
L_{2}(q)=\sum_{k_{1} \mid q} \sum_{k_{2} \mid q} \operatorname{gcd}\left(k_{1}, \frac{q}{k_{2}}\right)
$$

and

$$
L_{3}(q)=\sum_{k_{1} \mid q} \sum_{k_{2} \mid q} \sum_{k_{3} \mid q} \operatorname{gcd}\left(k_{1}, \frac{q}{k_{3}}\right) \operatorname{gcd}\left(k_{2}, \frac{q}{k_{3}}\right) \operatorname{gcd}\left(k_{1}, \frac{q}{k_{2}}\right) .
$$

We shall prove formula (8) first. We fix an $x_{1}, x_{2}$ Cartesian coordinate system in $\mathbf{R}^{2}$. Given any 2-dimensional $\bmod q$ lattice $\Lambda$, we have a basis-free representation for it as follows: The $x_{1}$ axis contains infinitely many points of $\Lambda$, with a density $1 / k_{1}$, where $k_{1}$ is a positive integer which divides $q$. Every line $x_{2}=c$ either contains no points of $\Lambda$ or contains a shifted copy of the set of lattice points on $x_{2}=0$. If $x_{2}=k_{2}$ is the line $x_{2}=c>0$ which is closest to the $x_{1}$ axis and has points of $\Lambda$, then $k_{2}$ is a divisor of $q$. A line $x_{2}=c$ contains points of $\Lambda$ if and only if has the form $x_{2}=t k_{2}$ for some integer $t$. We say that $\Lambda$ has jump $k_{2}$ (in the $x_{2}$ direction). If we 
let $C_{2}(\Lambda)$ denote the 2-dimensional volume of a fundamental cell of $\Lambda$, then we have $C_{2}(\Lambda)=k_{1} k_{2}$.

To count the 2-dimensional $\bmod q$ lattices which have given values of $k_{1}$ and $k_{2}$, it suffices to count the number of distinct 1-dimensional sublattices on $x_{2}=k_{2}$ which give a $\bmod q$ lattice. We define the shift $s$, where $s$ is an integer such that $0 \leq s<k_{1}$, to be the amount by which the 1-dimensional sublattice on $x_{2}=k$ is shifted with respect to the 1 -dimensional sublattice on $x_{2}=0$. In order to give a $\bmod q$ lattice, the shift $s$ must give a 1-dimensional sublattice on $x_{2}=q$ which is an unshifted copy of the same sublattice on $x_{2}=0$. The sublattice on $x_{2}=q$ is shifted from the one on $x_{2}=0$ by $q s / k_{2}$, so the shift $s$ gives a mod $q$ lattice if and only if

$$
k_{1} \text { divides } q s / k_{2} \text {. }
$$

Clearly (10) holds for given $k_{1}$ and $k_{2}$ if and only if $k_{1} k_{2} / \operatorname{gcd}\left(k_{1} k_{2}, q\right)=D$, say, divides $s$. Thus there are $k_{1} / D=\operatorname{gcd}\left(k_{1}, q / k_{2}\right)$ allowable values of $s$ in the range $0 \leq s<k_{1}$. This proves (8).

Now we prove formula (9). Each 3 -dimensional $\bmod q$ lattice $\Lambda$ is made up of a 2-dimensional $\bmod q$ sublattice in the $x_{1}, x_{2}$ plane, which we denote by $P_{0}$, and shifted copies of this sublattice in each of various planes $P_{i}(i$ nonzero integer) which are equally spaced parallel to $P_{0}$. As before, we let $1 / k_{1}$ denote the density of the points of $\Lambda$ on the $x_{1}$ axis and we let $k_{2}$ denote the jump in the $x_{2}$ direction for the sublattice in $P_{0}$ (and so for $\Lambda$ ). The plane $P_{1}$ nearest to $P_{0}$ is at a distance $k_{3}$, where $k_{3}$ is a divisor of $q$. We say that $\Lambda$ has jump $k_{3}$ in the $x_{3}$ direction. If we let $C_{3}(\Lambda)$ denote the 3 -dimensional volume of a fundamental cell of $\Lambda$, then we have $C_{3}(\Lambda)=k_{1} k_{2} k_{3}$.

To count the 3 -dimensional $\bmod q$ lattices with given $k_{1}, k_{2}$ and $k_{3}$, for each 2 dimensional $\bmod q$ sublattice on $P_{0}$ we count the number of distinct 2-dimensional sublattices in $x_{3}=k_{3}$ (i.e., the plane $P_{1}$ ) which give a mod $q$ lattice. We let $s$ denote the shift for the 1-dimensional sublattices in $P_{0}$, as before, and we define the (vector) shift $\mathbf{s}=\left(s_{1}, s_{2}\right)$, where $0 \leq s_{i}<k_{i}(i=1,2)$, to be the amount by which $\mathbf{0}$ in $P_{0}$ is moved when we go to the sublattice in $P_{1}$. The shift $\mathbf{s}$ gives a $\bmod q$ lattice if and only if

$$
k_{1} \text { divides } q s_{1} / k_{3} \text { and } k_{2} \text { divides } q s_{2} / k_{3},
$$

that is, if and only if the orthogonal projection of $\left(q / k_{3}\right)\left(s_{1}, s_{2}, k_{3}\right)$ into the plane $P_{0}$ is a lattice point. Now (11) holds for given $k_{1}, k_{2}$ and $k_{3}$ if and only if $k_{i} k_{3} / \operatorname{gcd}\left(k_{i} k_{3}, q\right)=$ $D_{i}$, say, divides $s_{i}(i=1,2)$. Thus there are $k_{i} / D_{i}=\operatorname{gcd}\left(k_{i}, q / k_{3}\right)$ allowable values of $s_{i}$ in the range $0 \leq s_{i}<k_{i}$. This proves (9).

It is possible to extend the formula in Theorem 2 to the case of general $m$, but complicated $m$-fold sums are involved. Since we do not need this result, we do not give it here.

A multiplicative function is completely determined by its values at prime powers, so it is of interest to examine $L_{m}\left(p^{a}\right)$ for prime $p$. Direct calculation using (8) gives

$$
L_{2}\left(p^{a}\right)=\sum_{i=0}^{a}(1+2 i) p^{a-i}=\frac{(p+1) p^{a+1}-(2 a+3) p+2 a+1}{(p-1)^{2}} .
$$


Computer calculations using (9) give Table 1, which shows the expansion of $L_{3}\left(p^{a}\right)$ in powers of $p$ for small $a$. There does not seem to be any nice explicit formula for $L_{3}\left(p^{a}\right)$, though various properties of the coefficients in the table can be deduced. Table 2 gives some values for $L_{2}(q)$ and $L_{3}(q)$.

\begin{tabular}{|c|c|c|c|c|c|c|c|c|c|c|c|c|c|c|c|}
\hline$a, j \rightarrow$ & 0 & 1 & 2 & 3 & 4 & 5 & 6 & 7 & 8 & 9 & 10 & 11 & 12 & 13 & 14 \\
\hline 1 & 4 & 2 & 2 & & & & & & & & & & & & \\
\hline 2 & 7 & 6 & 6 & 5 & 3 & & & & & & & & & & \\
\hline 3 & 10 & 10 & 12 & 10 & 10 & 8 & 4 & & & & & & & & \\
\hline 4 & 13 & 14 & 18 & 17 & 18 & 14 & 15 & 11 & 5 & & & & & & \\
\hline 5 & 16 & 18 & 24 & 24 & 28 & 22 & 24 & 20 & 20 & 14 & 6 & & & & \\
\hline 6 & 19 & 22 & 30 & 31 & 38 & 32 & 35 & 30 & 30 & 27 & 25 & 17 & 7 & & \\
\hline 7 & 22 & 26 & 36 & 38 & 48 & 42 & 48 & 42 & 42 & 38 & 38 & 34 & 30 & 20 & 8 \\
\hline
\end{tabular}

Table 1: Coefficients of $p^{j}$ in the expansion of $L_{3}\left(p^{a}\right), a \leq 7$.

\begin{tabular}{|c|c|c|c|c|c|c|c|c|c|c|c|c|c|}
\hline & 2 & 3 & 4 & 5 & 7 & 8 & 9 & 11 & 13 & 16 & 17 & 19 & 23 \\
\hline$L_{2}(q)$ & 5 & 6 & 15 & 8 & 10 & 37 & 23 & 14 & 16 & 83 & 20 & 22 & 26 \\
\hline$L_{3}(q)$ & 16 & 28 & 131 & 64 & 116 & 830 & 457 & 268 & 368 & 4633 & 616 & 1016 & 1108 \\
\hline
\end{tabular}

Table 2: Values of $L_{2}(q)$ and $L_{3}(q)$ for small prime powers $q$.

\section{The connection with Galois numbers}

Because of (2), our next theorem shows that $L_{m}(q)=G_{m}(q)$ whenever $q$ is a prime.

Theorem 3. For any prime q, we have

$$
L_{m}(q)=\sum_{r=0}^{m} E(r, m, q)
$$

Proof. We have already seen that every $m$-dimensional mod $q$ lattice is the solution space of some system (4), where $A$ is an $m$ by $m$ matrix over the integers mod $q$. Conversely, the solution space of any system (4) is an $m$-dimensional $\bmod q$ lattice. Since $q$ is prime, the mod $q$ lattices are thus in one-to-one correspondence with the $m$ by $m$ reduced row echelon forms of matrices over $G F(q)$ and we have the desired equation.

Because of (3), it is easy to compute $E(r, m, q)$ for given values of $r, m, q$.

If $q$ is not prime, the first two sentences in the proof of Theorem 3 are still true, so the one-to-one correspondence between the $\bmod q$ lattices and solution spaces of systems (4) is still valid. What is lost is the link with matrices over a field which 
are in reduced row echelon form (rref). Thus this paper shows that there are two different natural extensions of the Galois numbers $G_{n}(q), q$ prime. One extension leads to the Galois numbers $G_{n}(q)$ for arbitrary positive integers $q$, as given in [4]. In that paper a formal definition of a rref matrix over a set of $q$ symbols is given and finite fields play no role. For each $n$, the numbers $G_{n}(q)$ are fixed polynomials in $q$, and the recursion (1) holds as a polynomial identity. The other extension leads to the multiplicative functions $L_{n}(q)$ in this paper. If $q$ is not prime, then $L_{n}(q)$ is not a polynomial in $q$ and the analog of (1) does not hold.

\section{References}

[1] Miklos Ajtai, Generating hard instances of lattice problems, in: Proc. 28th ACM Symposium on the Theory of Computing, 1996, pp. 99-108.

[2] Thomas W. Cusick, The Ajtai random class of lattices, to appear.

[3] Jay Goldman and Gian-Carlo Rota, The number of subspaces of a vector space, in: Recent Progress in Combinatorics, ed. W. T. Tutte (Academic Press, 1969), pp. $75-83$.

[4] Albert Nijenhuis, Anita E. Solow and Herbert S. Wilf, Bijective methods in the theory of finite vector spaces, J. Combin. Theory (A) 37 (1984), 80-84. 